\title{
Magnetic properties of sputtered Permalloy/molybdenum multilayers
}

\author{
M. Romera, ${ }^{1, a)}$ R. Ranchal, ${ }^{2}$ D. Ciudad,${ }^{1}$ M. Maicas, ${ }^{1}$ and C. Aroca ${ }^{1}$ \\ ${ }^{1}$ ISOM \& Dpto. Física Aplicada, E.T.S.I. Telecomunicación, Universidad Politécnica de Madrid, \\ Ciudad Universitaria s/n, Madrid 28040, Spain \\ ${ }^{2}$ Dpto. Física de Materiales, Facultad CC. Físicas, Universidad Complutense de Madrid, \\ Ciudad Universitaria s/n, Madrid 28040, Spain
}

(Received 10 June 2011; accepted 31 August 2011; published online 20 October 2011)

\begin{abstract}
In this work, we report the magnetic properties of sputtered Permalloy $\left(\mathrm{Py}: \mathrm{Ni}_{80} \mathrm{Fe}_{20}\right)$ /molybdenum (Mo) multilayer thin films. We show that it is possible to maintain a low coercivity and a high permeability in thick sputtered Py films when reducing the out-of-plane component of the anisotropy by inserting thin film spacers of a non-magnetic material like Mo. For these kind of multilayers, we have found coercivities which are close to those for single layer films with no out-of-plane anisotropy. The coercivity is also dependent on the number of layers exhibiting a minimum value when each single Py layer has a thickness close to the transition thickness between Neel and Bloch domain walls. (C) 2011 American Institute of Physics. [doi:10.1063/1.3647768]
\end{abstract}

\section{INTRODUCTION}

The properties of magnetic materials for sensor applications must follow some general requirements like the linearity of the hysteresis loop, a high permeability, and a low coercive field. ${ }^{1}$ Permalloy (Py: $\mathrm{Fe}_{20} \mathrm{Ni}_{80}$ ) is a well known magnetic material characterized by its low saturating $\left(\mathrm{H}_{\mathrm{K}} \sim 5 \mathrm{Oe}\right)$ and coercive fields $\left(\mathrm{H}_{\mathrm{C}} \sim 1 \mathrm{Oe}\right)$ and can be grown with low internal stresses and nearly zero magnetostriction. ${ }^{2}$ These properties make Py a very interesting material for different applications, like anisotropic magnetoresistance sensors, ${ }^{3}$ magnetic recording heads, ${ }^{4}$ or flux concentrators for MEMS. ${ }^{5}$ Properties like permeability or linearity of the hysteresis loops can be controlled by growing Py as a sputtered multilayer with alternating perpendicular anisotropies. ${ }^{6}$ However, for thicknesses over $180 \mathrm{~nm}$, sputtered Py films develop perpendicular anisotropy (induced by the columnar morphology of the sputtered films) and these magnetic properties degrade notably. ${ }^{2}$ Both coercive and saturating fields are nearly 10 times bigger than those measured for thinner films. There are other growth techniques such as rapid quenching in which it is possible to obtain thick magnetic layers without out of plain anisotropy due to the absence of columnar morphology. However, those techniques do not allow to control essential properties like the magnetic anisotropy axis. In addition, the quality and structural properties of sputtered multilayers are desired in applications that need to integrate magnetic films on micro- or nanodevices. Therefore, the possibility of having thick sputtered Py films, while maintaining a low coercive field and a high permeability, would be of great interest in many applications. In this work, we study the magnetic properties of multilayer Py films with molybdenum spacers grown by magnetron sputtering.

\section{EXPERIMENTAL DETAILS}

Py/Mo multilayers were deposited by DC magnetron sputtering on thermally oxidized $\mathrm{Si}$ substrates with an $\mathrm{Ar}$

\footnotetext{
a) Author to whom correspondence should be addressed. Electronic mail: mromera@fis.upm.es.
}

pressure of $1.5 \times 10^{-3}$ mbar and a deposition power of $100 \mathrm{~W}$. These conditions have been chosen in order to minimize the stress induced during the growing process. ${ }^{2}$ Base pressure was lower than $10^{-6} \mathrm{mbar}$, and the distance from target to substrate was about $15 \mathrm{~cm}$. The growing rates were 0.14 and $0.03 \mathrm{~nm} / \mathrm{s}$ for Py and Mo, respectively. A vibrating sample magnetometer (VSM) and a magnetic force microscope (MFM) were used for the magnetic characterization of the samples, which was carried out at room temperature.

\section{RESULTS}

A single Py layer of $125 \mathrm{~nm}$ was grown to analyze the magnetic characteristics of a sputtered Py thin film. This thickness is below the critical value from which a perpendicular magnetic anisotropy is developed. Figure 1 shows the VSM hysteresis loops measured at different angles $\left(\alpha=0^{\circ}\right.$, $45^{\circ}, 90^{\circ}$ ) between the applied magnetic field and the easy axis of the in-plane magnetic anisotropy. The figure shows that the single Py layer has an anisotropy field of about 25 Oe and a coercive field of $0.6 \mathrm{Oe}$ in the hard axis and $1.5 \mathrm{Oe}$ in the easy axis. The resulting anisotropy, $\mathrm{K}=1200 \mathrm{~J} / \mathrm{m}^{3}$, corresponds to an exchange correlation length of $180 \mathrm{~nm}$. This Py sample was deposited using a sputter beam with an angle of incidence of about 25 degrees. Then, this film exhibits a magnetic anisotropy that is induced in the direction of incidence beam, being parallel to the intersection between the incidence and the substrate planes (Figure 1, inset) as described in previous works. $^{6-10}$

Samples with Py thicknesses of 125, 250, and $375 \mathrm{~nm}$ were grown in order to study the effect of the layer thickness on magnetic properties. Hysteresis loops for these samples are shown in Figure 2. The films develop an increase in the coercivity and saturating field when increasing the thickness. The shape of the magnetization curves reveals a change in the magnetic anisotropy from an in-plane anisotropy, for the thinner samples, to an out-of-plane anisotropy for the thicker samples.

Due to the "columnar" nature of the films growth, the thicker the sample is, the higher the perpendicular anisotropy 


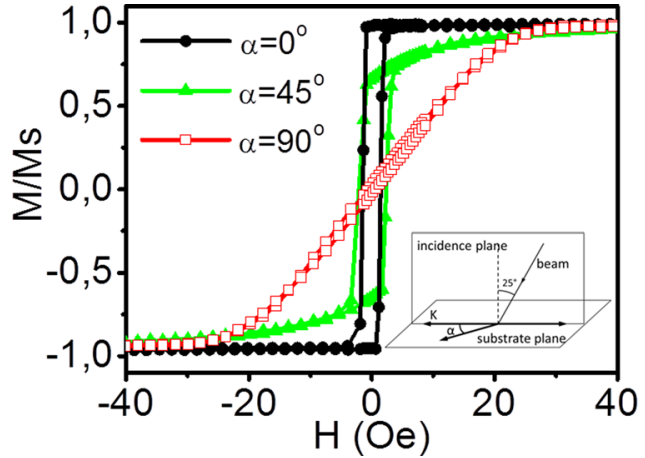

FIG. 1. (Color online) Hysteresis loop of a Py single layer with a thickness of $125 \mathrm{~nm}$, measured at $\alpha=0^{\circ}$ (filled circles), $\alpha=45^{\circ}$ (filled triangles), and $\alpha=90^{\circ}$ (empty squares) between the applied magnetic field and the direction of the easy axis.

induced. ${ }^{7,11}$ At a certain thickness, this anisotropy makes the magnetization have a component perpendicular to the surface, and the sign of this magnetization component alternates from one domain to the next in order to reduce the magnetostatic energy. As a result, thick layers need much higher fields to saturate the in-plane hysteresis loops $(\sim 100 \mathrm{Oe})$ (Figure 2(c)) than thin films (Figures 1 and 2(a)). Coercive fields also increase with thickness measuring values around 25 Oe for thicknesses above $180 \mathrm{~nm}^{2}$ MFM measurements show a stripe domain pattern that proves the existence of an anisotropy component perpendicular to the sample plane (Figure 3).

In order to break this perpendicular anisotropy, thin Mo interlayers were introduced in the structure. The thickness of the Mo spacer was chosen large enough to avoid any exchange coupling between the Py magnetic layers. To check that, we grew a $\operatorname{Py}(125 \mathrm{~nm}) / \mathrm{Mo}(10 \mathrm{~nm}) / \operatorname{Py}(125 \mathrm{~nm})$ trilayer. The anisotropy axis in the Py layers is in the in-plane direction being perpendicular to each other (Figure 4, inset). This anisotropy configuration is achieved by controlling the direction of the incidence beam. First Py layer is deposited with the sample holder in one fixed position and then it is rotated $90^{\circ}$ before growing the second Py layer as described in previous works. ${ }^{6,9}$ Due to the symmetry the hysteresis loop (Figure 4) is similar in the two perpendicular anisotropy axis of this sample and it can be considered as the addition of two loops, one for the easy axis and another for the hard axis. This agrees with two Py layers magnetically isolated with independent magnetic processes.

Figure 5 shows the magnetic behavior of a Py/Mo multilayer consisting in 4 Py layers of $62.5 \mathrm{~nm}$ for a total Py thickness of $250 \mathrm{~nm}$. If we compare the loop for this $\mathrm{Py} / \mathrm{Mo}$

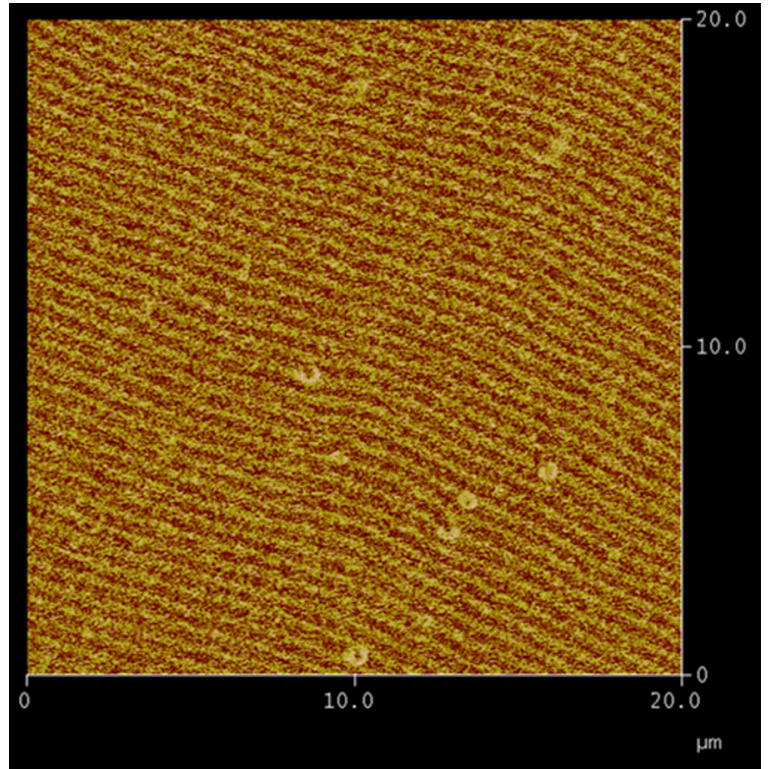

FIG. 3. (Color online) MFM image of the magnetic stripe domains in the Py single layer sample with a thickness of $375 \mathrm{~nm}$.

multilayer with that for a single Py layer with the same total thickness (Figure 2(b)), we observe that the multilayer has lower saturating and coercive fields. Moreover, $\mathrm{H}_{\mathrm{K}}$ and $\mathrm{H}_{\mathrm{C}}$ are even lower than those measured for the single Py layer with a lower thickness (Figure 1). These values and the shape of the hysteresis loop indicate that we have actually broken the perpendicular anisotropy.

The influence of the number of layers on the coercive field of Py/Mo multilayers with a total constant Py thickness of $250 \mathrm{~nm}$ is shown in Figure 6. These coercive fields were obtained from hysteresis loops recorded with the magnetic field applied in both the hard and easy axis direction. In these multilayers, the anisotropy axis is in the plane and it has the same direction in every layer. As the total Py thickness is constant, the increase in the number of layers is associated to a decrease in the thickness of each Py layer. These Py layers are separated by Mo spacers with a constant thickness of $10 \mathrm{~nm}$ each. Results show a decrease in coercivity when the number of layers increases from 4 to 8 , which is associated to a change in the domain wall structure from Bloch to Nèel walls. In the 8 layers sample, the thickness of each Py layer is $t_{\mathrm{m}}=30 \mathrm{~nm}$. The transition between Nèel and Bloch walls in Py occurs at a thickness around $50 \mathrm{~nm}$, Nèel walls for layers below this thickness and Bloch walls for layers above this thickness. Then, it is expected that the sample with 4 layers has Bloch-type domain walls while the sample with 8

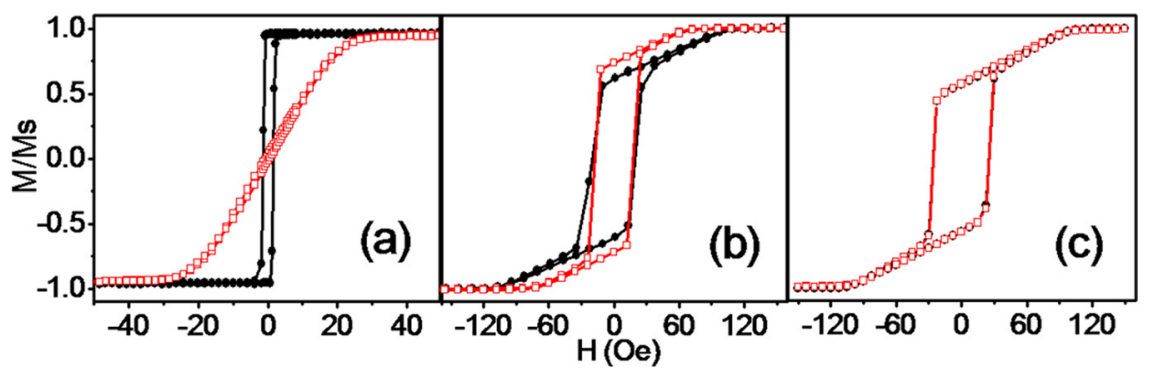

FIG. 2. (Color online) Hysteresis loops at the easy (filled circles) and hard axis (empty squares) of the in-plane magnetic anisotropy, for Py single layers of (a) 125 , (b) 250 , and (c) $375 \mathrm{~nm}$, respectively. 


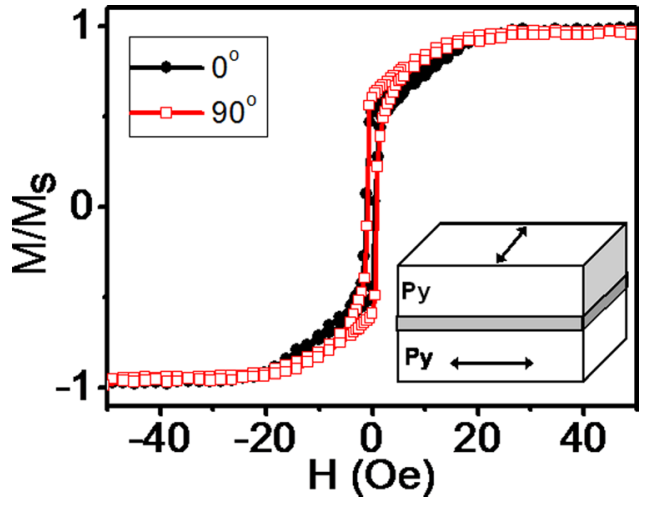

FIG. 4. (Color online) Hysteresis loops of a trilayer Py $(125 \mathrm{~nm}) / \mathrm{Mo}$ $(10 \mathrm{~nm}) / \mathrm{Py}(125 \mathrm{~nm})$ with perpendicular anisotropies, measured at two perpendicular directions corresponding to the in-plane anisotropy axis of the Py layers. Inset: Schematic representation of the anisotropy configuration of the trilayer.

Py layers has Nèel-type domain walls. Nèel type walls are wider than Bloch walls so the pinning with defects is expected to be lower and thus the measured coercivity.

Multilayers with more than 8 Py layers show an increase of the coercivity as the number of layers increases. This result is in contrast with the effect of thickness in Py single layers where other authors have found a decrease in the coercivity with the decrease of the layer thickness in the range between 30 and $7 \mathrm{~nm} .^{12}$ In the case of our multilayers, it is necessary to consider two new effects when comparing with Py single layers: the magnetostatic coupling between domain walls in different layers and the increase in the number of boundary surfaces. Nèel walls may induce quasi-domainwalls in the contiguous coupled layer due to the magnetostatic coupling, and so, domain wall dynamics is affected by them. ${ }^{13,14}$ Quasi-domain walls are not moved by the applied field so they hamper the free displacement of the coupled domain walls. In addition, a large number of boundaries between Py and Mo contributes also to increase the surface effects on coercive field. These two effects explain the increase of coercivity when the Py thickness layer is reduced. In any case, all measured coercive fields were below $2 \mathrm{Oe}$, which are much lower than those measured for the single 250 Py film $(\mathrm{Hc} \sim 20 \mathrm{Oe})$.

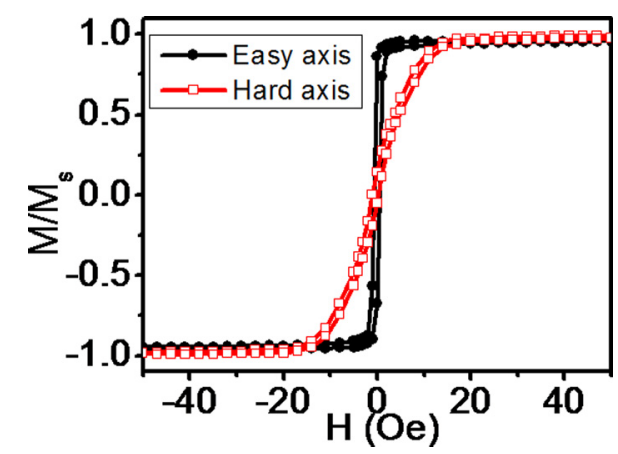

FIG. 5. (Color online) Hysteresis loops of a Py/Mo multilayer with 4 Py layers recorded with the applied magnetic field in both the easy (filled circles) and the hard axis (empty squares) of the in-plane magnetic anisotropy. The total Py thickness is $250 \mathrm{~nm}$.

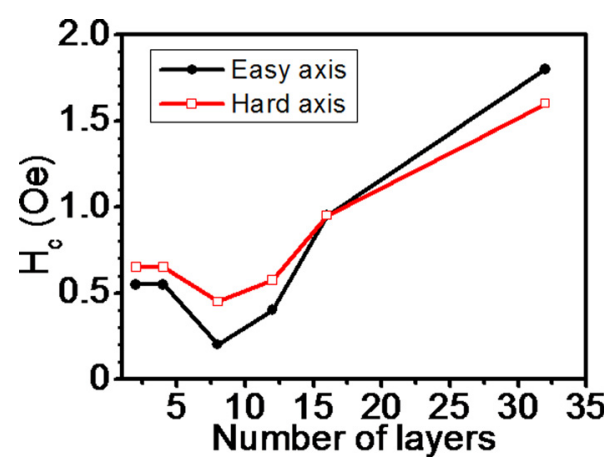

FIG. 6. (Color online) Coercive field of Py/Mo multilayers with a total Py thickness of $250 \mathrm{~nm}$ as a function of the number of Py layers. Hysteresis loops were recorded with the applied magnetic field in both the easy (filled circles) and the hard axis (empty squares) of the in-plane magnetic anisotropy.

\section{CONCLUSIONS}

In this work, we show that it is possible to reduce the perpendicular anisotropy component in thick sputtered Py films by introducing Mo spacers. We have found a minimum coercivity for these Py/Mo multilayers when the Py thickness is close to the transition between Nèel- and Bloch-type walls. The increase of the coercive field in multilayers with Py layers below this transition thickness has been related to the DWs magnetostatic interaction as well as surface effects. The possibility of maintaining in-plane anisotropy on sputtered Py/Mo multilayers with a total Py thickness higher than $180 \mathrm{~nm}$ allows us to keep low coercive and saturating fields. The coercivities are 1 order of magnitude lower than that measured in single Py films with the same thickness.

\section{ACKNOWLEDGMENTS}

The work has been financially supported by projects MAT2007-65965-C02 (MEC), SAL-0312/2006 (CAM) and CCG10-UCM/MAT-4621 (CAM and Universidad Complutense de Madrid). We acknowledge the Spanish Ministerio de Ciencia e Innovación for funding M. Romera through the FPU fellowship AP2007-00464.

${ }^{1}$ P. Ripka, Magnetic Sensors and Magnetometers (Artech House, Boston, 2001), p. 88.

${ }^{2}$ S. F. Cheng, P. Lubitz, Y. Zheng, and A. S. Edelstein, J. Magn. Magn. Mat. 282, 109 (2004).

${ }^{3}$ H. Hauser, J. Hochreiter, G. Stangl, R. Chabicovsky, M. Janiba, and K. Riedling, J. Magn. Magn. Mat. 215, 788 (2000).

${ }^{4}$ J. McCord and J. Westwood, IEEE Trans. Magn. 37, 1755 (2001).

${ }^{5}$ A. S. Edelstein and G. A. Fisher, J. Appl. Phys. 91, 7795 (2002).

${ }^{6}$ M. Maicas, R. Ranchal, C. Aroca, P. Sánchez, and E. López, Eur. Phys. J. B 62, 267 (2008).

${ }^{7}$ K. Hara, T. Hashimoto, and E. Tatsumoto, J. Phys. Soc. Japan, 28, 254 (1970).

${ }^{8}$ E. W. Pugh, E. L. Boyd, and J. F. Freedman, IBM J. Res. Dev. 4, 163 (1960).

${ }^{9}$ M. González-Guerrero, J. L. Prieto, P. Sánchez, and C. Aroca, J. Appl. Phys. 102, 123903 (2007).

${ }^{10}$ R. E. Jones, J. Williams, L. Spector, C.-J. Lin, S. Wang, S. Pichai, and B. M. Clemens, IEEE Trans. Magn. 31, 3817 (1995).

${ }^{11}$ D. O. Smith, M. S. Cohen, and G. P. Weiss, J. Appl. Phys. 31, 1755 (1960).

${ }^{12}$ M. A. Akhter, D. J. Mapps, Y. Q. Ma. Tan, A. Petford-Long, and R. Doole, J. Appl. Phys. 81, 4122 (1997).

${ }^{13}$ M. Maicas, M. A. Rivero, E. López, M. C. Sánchez, C. Aroca, and P. Sánchez, J. Magn. Magn. Mater. 203, 289 (1999).

${ }^{14}$ R. Ranchal, C. Aroca, and E. López, New J. Phys. 10, 013013 (2008). 